\title{
The SIMBAD astronomical database
}

\section{The CDS reference database for astronomical objects}

\author{
M. Wenger, F. Ochsenbein, D. Egret, P. Dubois, F. Bonnarel, S. Borde ${ }^{\star}$, F. Genova, G. Jasniewicz ${ }^{\star \star}$, S. Laloë, \\ S. Lesteven, and R. Monier
}

CDS, Observatoire astronomique de Strasbourg, UMR 7550, 11 rue de l'Université, F-67000 Strasbourg, France

Received December 1, 1999; accepted January 17, 2000

\begin{abstract}
SimBAD is the reference database for identification and bibliography of astronomical objects. It contains identifications, "basic data", bibliography, and selected observational measurements for several million astronomical objects.

SIMBAD is developed and maintained by CDS, Strasbourg. Building the database contents is achieved with the help of several contributing institutes. Scanning the bibliography is the result of the collaboration of CDS with bibliographers in Observatoire de Paris (DASGAL), Institut d'Astrophysique de Paris, and Observatoire de Bordeaux.

When selecting catalogues and tables for inclusion, priority is given to optimal multi-wavelength coverage of the database, and to support of research developments linked to large projects. In parallel, the systematic scanning of the bibliography reflects the diversity and general trends of astronomical research.

A WWW interface to SIMBAD is available at:

http://simbad.u-strasbg.fr/Simbad.
\end{abstract}

Key words: astronomical data bases: miscellaneous catalogs

\section{Introduction}

\subsection{The $C D S$}

The Centre de Données astronomiques de Strasbourg (CDS) defines, develops, and maintains services to help the astronomers find the information they need from the

Send offprint requests to: D. Egret,

e-mail: question@simbad.u-strasbg.fr

* DASGAL, Observatoire de Paris.

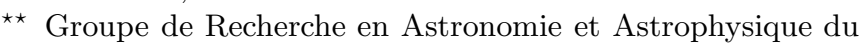
Languedoc (GRAAL), Montpellier. very rapidly increasing wealth of astronomical information, and particularly of on-line information.

CDS is operated at the Strasbourg astronomical Observatory, under an agreement between French Institut National des Sciences de l'Univers (INSU) and Université Louis Pasteur, Strasbourg (ULP). CDS personnel created and implemented the SIMBAD data bank and maintain its data and software system.

A detailed description of the CDS on-line services can be found, e.g., in Egret et al. (1995) and in Genova et al. (1996, 1998, 2000), or at the CDS web site ${ }^{1}$. Questions or comments about the CDS services can be sent to the hot linequestion@simbad.u-strasbg.fr.

\section{2. $S I M B A D$}

The Simbad database contains identifications, "basic data", bibliographical references, and selected observational measurements for more than 2.7 million astronomical objects (November 1999). Data and information published in SIMBAD come from selected catalogues and tables and from the whole astronomical literature.

The specificity of the SIMBAD database is to organize the information per astronomical object, thus offering a unique perspective on astronomical data. This is done through a careful cross-identification of objects from catalogues, lists, and journal articles. The ability to gather together any sort of published observational data related to stars or galaxies has made SIMBAD a key tool used worldwide for all kinds of astronomical studies.

SIMBAD is the acronym for Set of Identifications, Measurements and Bibliography for Astronomical Data.

The main access point to SIMBAD is the WWW home page $^{2}$; there is a mirror copy at SAO, Harvard ${ }^{3}$.

\footnotetext{
${ }^{1}$ Internet address: http://cdsweb.u-strasbg.fr/

2 http://simbad.u-strasbg.fr/Simbad

3 http://simbad.harvard.edu/Simbad
} 


\subsection{Historical background}

Building a reference database for stars - and, later, for extragalactic objects and all astronomical objects outside the Solar System - has been the first goal of the CDS: SIMBAD is the result of an on-going effort which started soon after the creation of CDS in 1972. SIMBAD was created by merging the catalog of Stellar Identifications (CSI, Ochsenbein et al. 1981) and the Bibliographic Star Index (Ochsenbein 1982) as they existed until 1979. The resulting data base (at that time, about 400000 objects, mainly stars) was then expanded by the addition of source data from the catalogs and tables, and by new literature references. The database was extended to galaxies and other non-stellar objects in 1983 (Dubois et al. 1983). For details about the early developments of SIMBAD see Egret (1983).

The first on-line interactive version of SIMBAD was released in 1981, and operated at the Strasbourg Cronenbourg computer center until December 1984, when it was moved to Université Paris-Sud at Orsay, and operated there until June 30, 1990. The database is now hosted on a Unix server, at the Strasbourg Observatory.

The original command line interface has been complemented by an interactive X-Window interface (XSIMBAD) in 1994, and by a World-Wide Web interface in 1996. There is also a client/server mode, providing quick responses to simple queries, essentially for the name resolution in archives and information systems (see Sect. 5).

For descriptions of earlier stages of the database, see Heck \& Egret (1986), and Egret et al. (1991).

\section{SIMBAD main features}

SIMBAD is, in the first place, a database of identifications, aliases and names of astronomical objects: in principle any name found in the literature - provided it is given as a syntactically correct character string - can be submitted to SIMBAD in order to retrieve basic information known for this object, as well as pointers to complementary data and bibliography. This implies a continuous careful crossidentification of objects from catalogues, lists, and journal articles. This ability to gather together any sort of published observational data related to stars or galaxies is the first key feature of SIMBAD.

Another unique feature is the complete and up-to-date bibliographic survey of the astronomical literature: objects are associated with the references of all papers in which they are mentioned, independently of the aliases used to name the object.

In addition, the Dictionary of Nomenclature (Sect. 8) is an essential tool for managing the very complex nomenclature of objects found in the literature, and for matching naming variations with those adopted or simply accepted by SImBAD. It also includes hints for helping to solve ambiguities, according to the type of object, or to the format. This is complemented by the sesame module within SIMBAD, for the management of possible variations in the naming of astronomical objects.

The database management system of SIMBAD (Sect. 4) has been developed in-house at CDS, using the concepts of object-oriented programming.

SIMBAD is kept up-to-date (Sect. 7) on a daily basis, as the result of the collaboration of CDS with bibliographers in Institut d'Astrophysique de Paris and the Paris and Bordeaux observatories.

The statistical contents of the database (Sect. 3) can be summarized in a few figures as follows (the figures quoted are statistics of November 1999):

- entries for about 2.7 million astronomical objects (stars, galaxies and all astronomical objects outside the solar system);

- a cross-index of 7.5 million identifiers related to 4500 astronomical catalogues and tables, lists, and observation logs of space missions;

- observational data from some 25 different types of data catalogues and compilations;

- a bibliographic survey covering the astronomical literature since 1950 for stars, and since 1983 for extragalactic objects: more than 3 million citations from 110000 different papers.

\section{SIMBAD astronomical contents}

\subsection{The objects}

The SimbaD data base presently contains information for about:

- 1500000 stars;

- 450000 galaxies;

- 100000 other non-stellar objects (planetary nebulae, clusters, H II regions, etc.);

- and some 650000 additional objects observed at various wavelengths (Radio, IR, X), and for which classification is not yet assigned.

The only astronomical objects specifically excluded from SimBAD are the Sun and Solar System bodies.

The SImBAD database is primarily organized per astronomical object. The aim is to provide, as much as possible, the user with all published information (identifications, observational data, bibliographical references, and pointers towards external archives) concerning any given object, or list of objects.

The two main channels for feeding the database are the following:

- the daily scanning of papers published in the astronomical literature provides new references and new identifiers for existing objects, as well as opportunities 
to create new objects, using the basic data possibly given in the article;

- the complete (or partial) folding of selected catalogues into the database serves as a basis for improving the completeness and multi-wavelength coverage of the database.

Catalogues are selected for integration with priority given to those which can help provide an optimal support for the large projects conducted within the astronomical community. A large effort was, for instance, devoted in recent years to stellar catalogues (PPM, HIC, CCDM), in the context of the Hipparcos project, and to multi-wavelength identifications (IRAS PSC, Einstein $1 \mathrm{E}$ and $2 \mathrm{E}$ catalogues, older X-ray catalogues, the IUE Merged Log, etc.). The Hipparcos and Tycho catalogues (ESA 1997) have been recently included, and inclusion of the ROSAT All Sky Survey is planned in the near future.

In parallel, the systematic scanning of the bibliography reflects the diversity and general trends of research in astronomy, and takes into account shorter lists. The published lists from the microlensing surveys, or e.g. the EUVE catalogues, were folded into the database as a result of this scanning.

When an object is found in the literature or in a catalogue, its possible cross-identification with objects already in SImBAD is systematically studied, before entering the reference and the new object name in the database. About 4500 different names of catalogues or object lists from published papers can currently be found in SIMBAD, covering all the wavelength domains from high energy astrophysics to radio.

When no proper name is suggested by the authors, or when the acronym generates an ambiguity with already existing ones, the current practice, shared with the NED database, is to create an acronym within brackets using the initials of the last names of the first three authors, and the year of publication. For example, [HFE83] 366 is the 366th entry in the main table of a paper by Helmer, Fabricius, Einicke and colleagues published in 1983. From the year 2000 on, the year will be noted with four digits (e.g., [ABC2000]).

Many objects have more than one name, since the database contains more than 7.5 million object names for 2.7 million objects. Examples of objects with more than 50 identifiers, are the galaxy M 87 in Virgo, the bright stars Procyon and Capella, the quasar 3C 273, the Crab Nebula.

To help the users with the complex nomenclature of astronomical objects, the CDS now maintains and distributes on-line the Dictionary of Nomenclature of Celestial Objects (first developed by Lortet et al. 1994; see Sect. 8).

\subsection{The data}

In the following, the word object will be used to designate a star, non stellar object, or collection of objects such as a cluster, which corresponds to an individual entry in SIMBAD. For each object, the following data are included when available:

- Basic data:

stars: object type, coordinates, proper motion, $B$ and $V$ magnitudes, spectral type, parallax, radial velocity;

galaxies: object type, coordinates, blue and visual integrated magnitudes, morphological type, dimension, radial velocity or redshift.

other: object type, position, $B$ and $V$ magnitudes.

- Cross-identifications from some 4500 catalogues and tables, either completely or partially included in the data base.

- Observational data (also called measurements), for some 25 data types. A list of these types is given in the Table 2.

- General bibliography, including references to all published papers since 1983 citing the object under any of its designations. For stars, the bibliography starts as early as 1950, but with a smaller coverage of the literature. SIMBAD also includes a few hundred references before 1950, but without any systematic trend. The bibliography gives access to abstracts and electronic articles when available (either directly from publishers, or through ADS). Currently about 100 journals covering the complete astronomical literature are regularly scanned. A complete list is available on line ${ }^{4}$.

In the following, a more detailed description of some of these elements is given.

\subsubsection{Object type}

The object type refers to a hierarchical classification of the objects in SIMBAD, derived by the CDS team on the basis of the catalogue identifiers (as proposed by Ochsenbein $\&$ Dubois 1992). From Star to Maser source, or Cluster of Galaxies, some 70 different categories, general, or very specific, are proposed (see examples in Table 1). A complete list is available on line ${ }^{5}$.

This classification is intended to help the user select objects out of the database (e.g. through the filter procedure, see Sect. 4.5). It is also a powerful tool for data cross-checking and quality control. It has been designed to be practical and useful, and complements other features also available in SIMBAD (morphological type or spectral type information, catalogues, and measurements). It can

\footnotetext{
4 http://simbad.u-strasbg.fr/guide/chH.htx

5 http://simbad.u-strasbg.fr/guide/chF.htx
} 
follow the evolution of astronomy, with the introduction of new categories recently appeared in the literature (e.g., in the last years, Low-Mass or High-Mass X-Ray binary, Microlensing event, or Void).

Each class has normally a standard designation, a condensed one (used in tables) and an extended explanation. The classification uses a hierarchy with four levels, reflecting our knowledge of the characteristics of the astronomical object. For instance, an object can be classified as a "Star" (this is level 1). If photometric observations have shown variability of the object, it can be classified as a "Variable star" (this is level 2). Examples of level 3 and 4 are "Pulsating variable", and "Cepheid".

This hierarchy of object types (and their possible synonyms) is managed in the database in such a way that selecting variable stars $(\mathrm{V} *)$ is understood as selecting objects classified as V*, and all subdivisions (e.g. PulsV*, Mira, Cepheid, etc.). If the user is only interested in RR Lyrae type stars, he/she will use the RRLyr type, leaving aside all other variable stars for which the variability mode is different, or not known.

The classification emphasizes the physical nature of the object rather than a peculiar emission in some region of the electromagnetic spectrum or the location in peculiar clusters or external galaxies. Therefore objects are classified as peculiar emitters in a given wavelength (such as UV or IR source) only if nothing more about the nature of the object is known, i.e. it cannot be decided on the sole basis of the basic data whether the object is a star, a multiple system, a nebula, or a galaxy. For instance, if an object appears only in the IRAS catalogue, it is automatically classified as IR object: it is left to the user to decide to go further and to derive, e.g. on the basis of the IRAS colors, the probability for the source to be stellar or extragalactic.

Because there is at most one object type per object, this classification should be used with caution when extracting samples out of the database. This is typically the case for the wavelength types: using IR or X as a criterion cannot generate a sample of all IRAS sources, or all $\mathrm{X}$-ray emitting objects, since a number of them are in fact classified as stars, galaxies, etc.

3.2.2. Coordinates, proper motion, parallax, and radial velocity or redshift

The coordinates were originally stored in the database in the FK4 system for equinox and epoch 1950.0. A major change was undergone in 1999, when they were moved to the International Celestial Reference System (ICRS, see Feissel \& Mignard 1998) at epoch 2000.0, after the publication of the Hipparcos and Tycho catalogues. The position data frame has become more complex, grouping together all data needed for computing the coordinates into any reference frame, at any epoch and equinox: the
Table 1. Object type classification: extracts from the object type table illustrating examples of the four levels of the classification scheme

\begin{tabular}{|c|c|c|c|}
\hline Level & $\begin{array}{l}\text { Standard } \\
\text { name }\end{array}$ & $\begin{array}{l}\text { Short } \\
\text { name }\end{array}$ & Extended Explanation \\
\hline$\cdots$ & Star & $*$ & Star \\
\hline $\begin{array}{l}1 \\
2\end{array}$ & $*_{\mathrm{inCl}}$ & $*_{\mathrm{iC}}$ & Star in Cluster \\
\hline 2 & $*_{\text {inNeb }}$ & $*_{\mathrm{iN}}$ & Star in Nebula \\
\hline 2 & $*_{\text {inAssoc }}$ & $*_{\mathrm{iA}}$ & Star in Association \\
\hline 2 & $*$ in $* *$ & $*_{\mathrm{i}} *$ & Star in double system \\
\hline 2 & $\mathrm{~V}^{*} ?$ & $\mathrm{~V}^{*} ?$ & Star suspected of Variability \\
\hline 2 & Pec* & $\mathrm{Pe}^{*}$ & Peculiar Star \\
\hline 3 & $\mathrm{HB}^{*}$ & $\mathrm{HB}^{*}$ & Horizontal Branch Star \\
\hline 3 & YSO & $\mathrm{Y}^{*} \mathrm{O}$ & Young Stellar Object \\
\hline 3 & Em* & Em* & Emission-line Star \\
\hline 4 & $\mathrm{Be}^{*}$ & $\mathrm{Be}^{*}$ & Be Star \\
\hline 1 & Galaxy & G & Galaxy \\
\hline 2 & PartofG & PoG & Part of a Galaxy \\
\hline 2 & $\mathrm{GinCl}$ & $\mathrm{GiC}$ & $\begin{array}{l}\text { Galaxy in Cluster of } \\
\text { Galaxies }\end{array}$ \\
\hline 2 & GinGroup & $\mathrm{GiG}$ & Galaxy in Group of Galaxies \\
\hline 2 & GinPair & $\mathrm{GiP}$ & Galaxy in Pair of Galaxies \\
\hline 2 & High_z_G & $\mathrm{HzG}$ & Galaxy with high redshift \\
\hline 2 & AGN & AGN & Active Galaxy Nucleus \\
\hline 3 & LINER & LIN & $\begin{array}{l}\text { LINER-type Active Galaxy } \\
\text { Nucleus }\end{array}$ \\
\hline 3 & Seyfert & SyG & Seyfert Galaxy \\
\hline 4 & Seyfert_1 & Sy1 & Seyfert 1 Galaxy \\
\hline 4 & Seyfert_2 & Sy2 & Seyfert 2 Galaxy \\
\hline 3 & Blazar & Bla & Blazar \\
\hline 4 & BLLac & BLL & BL Lac - type object \\
\hline 4 & OVV & OVV & Optically Violently Variable \\
\hline 3 & QSO & QSO & Quasar \\
\hline
\end{tabular}

coordinates themselves, the proper motion, the parallax and the radial velocity or redshift.

All these data contain the same subfields: the original data, displayed with a number of digits consistent with the announced precision of the data; a quality code from "A" (reference data) to "E" (unreliable origin); an error box (either a standard error, or an ellipse), and the bibliographic reference of the data.

In earlier versions of SIMBAD, the determination of the position for another equinox used to take only precession into account. In the current version, a change of equinox takes into account not only the precession but also the proper motion, the reference frame (FK4, FK5, ICRS), and, when they are known, the parallax and radial velocity. When no epoch is specified, the year of the equinox is used by default.

Data come from various sources. When astrometric data are available, the most accurate one has been selected for the "basic data". Other values may be available as measurements (in the pos type). The Hipparcos and 
Tycho catalogues (ESA 1997) constitute the major source of positions for stars.

The coordinates precision may vary from $1^{\circ}$ to $1 / 10$ mas. The default display format provides equatorial coordinates in the ICRS system at epoch 2000.0, and in the FK5 system at equinoxes 2000 and 1950, as well as galactic coordinates. Coordinates in the FK4 system, and ecliptic or super-galactic coordinates can be computed on request.

The proper motions $\left(\mu_{\alpha} \cos \delta, \mu_{\delta}\right)$ are given in mas/year, together with their standard errors (in mas/year). The primary source of proper motions is the Hipparcos and Tycho catalogues (ESA 1997).

The errors for positions or proper motions are expressed as error ellipses, made of three numbers, within brackets: the major axis, the minor axis, and the position angle of the major axis (measured from North to East). Major and minor axes are expressed in mas for the position, and mas/yr for the proper motion; the position angle is expressed in degrees, in the range $\left[0^{\circ}, 180^{\circ}[\right.$.

When available, the stellar parallax is given in mas, together with the associated error within brackets. The primary source is the Hipparcos and Tycho catalogues (ESA 1997).

Radial velocity (in $\mathrm{km} \mathrm{s}^{-1}$ ), or redshift (for extragalactic objects) are currently available for some 160000 objects. They are stored in their original type (either redshift, or radial velocity in $\mathrm{km} \mathrm{s}^{-1}$ ), associated with the standard error. Display can be done in the original type or forced to be one of the two types, using the corresponding translation formula.

Stellar radial velocity data have been compiled with the collaboration of Observatoire de Marseille.

For extragalactic objects, up-to-date redshift information has recently been imported from the NASA/IPAC Extragalactic Database (NED, Helou et al. 1991) as a result of the ongoing exchange agreement: the SIMBAD team is providing NED with bibliographic coverage of extragalactic objects for all astronomical journals, and is being given access, in return, to extragalactic data collected by NED.

Tables from individual articles constitute the other major source of information.

\subsubsection{Magnitudes}

$B$ and $V$ magnitudes are given, when possible, in the Johnson's $U B V$ system. Both magnitudes may be followed by a semicolon meaning they cannot be made homogeneous to the $U B V$ system. In addition the following flags may appear:

- a "D" flags a joint magnitude in a double or multiple system;

- a "V" indicates a variable magnitude and is followed by a coded index giving a rough estimate of the amplitude:

\begin{tabular}{cl}
\hline code & definition \\
\hline 1 & $1 / 100 \mathrm{mag}$ \\
2 & $1 / 10 \mathrm{mag}$ \\
3 & $1 \mathrm{mag}$ \\
4 & more than $1 \mathrm{mag}$ \\
$?$ & suspected variable \\
\hline
\end{tabular}

When possible the magnitudes have been taken from the Tycho Reference Catalogue (Høg et al. 1998) where $B$ and $V$ magnitudes are derived from the original $B_{\mathrm{T}}$ and $V_{\mathrm{T}}$. Another major source is the $U B V$ compilation of Mermilliod (1987). Otherwise the data would come from one of the published papers associated to the object.

\subsubsection{Stellar spectral type}

The spectral types of stars have been selected preferably in the Michigan Catalogues of Two-Dimensional Spectral Types for the HD stars (Houk 1975, and seq.), or in the bibliographical surveys of MK classifications (Jaschek 1978). In the absence of a full MK classification, the HD spectral type is recorded.

Most of the spectral types need less than 5 characters, but this field can be as long as 36 characters.

\subsubsection{Morphological type and dimension of galaxy}

The morphological types of galaxies have been selected primarily from the Uppsala General Catalogue of Galaxies (UGC, Nilson 1973), the Morphological Catalogue of Galaxies (MCG, Vorontsov-Velyaminov 1962), and other catalogues (see Dubois et al. 1983).

In complement, the following data, primarily from UGC, are given, when available, for galaxies:
$\log D_{25}$
logarithm of the major axis $a$ expressed in tenths of arc minutes;
$\log R_{25}$ logarithm of the ratio $a / b$ where $a$ and $b$ are the major and minor axis;
orientation orientation angle (in degrees); (inclination) inclination (in units of $15^{\circ}$ from 0 to 7 ).

\subsection{Cross-identifications}

\subsubsection{Aliases}

Cross-identifications of stars and galaxies have been searched for SIMBAD entries from (currently) about 4500 source catalogues and tables, included, either completely or partially, in the data base. The index of 7.5 million aliases, thus constituted, is one of the unique features of the SimBAD database. 
Aliases may serve as entry points for related catalogues and tables (e.g. in VIZIER). Cross-fertilization of a given research with previous studies of the same object published in the astronomical literature is made directly possible from the alias list.

The index of names and aliases constitutes the basis for the SIMBAD name resolver which provides, in response to any object name, the set of coordinates corresponding to the object position on the celestial sphere, or the list of papers citing the object. The name resolving power of SIMBAD is used by many archives and information systems (such as the archives of Hubble Space Telescope or European Southern Observatory, the High Energy Astrophysics Science Archive Center, the Astrophysics Data System, servers of the Digitized Sky Surveys, etc.).

There is no SIMBAD preferred name for objects ${ }^{6}$ : all aliases can be equally used. A short list of major catalogues is used internally to put at the top of the list the most common name according to the object type (e.g., Messier or NGC identifier for galaxies and nebulae). All other identifiers are presented in alphabetical order.

A command of the SIMBAD native node ("selectid"), and an option in the sampling form of the WWW interface, allow the user to impose a list of identifiers to be used when displaying object lists.

\subsubsection{Multiple systems}

It is to be noted that for a double system in which the components can be observed separately, SIMBAD frequently includes three entries: A and B components, and an additional entry for the joint system (AB), the latter entry carrying the observational data and references related to the system as a whole. This has to be taken into account in statistical studies such as stellar counts.

\subsection{Observational data}

Observational data are presently given for the measurement types listed in Table 2.

For each data type, one can retrieve individual data with their bibliographical references, and, when available, weighted means computed from existing observed values by specialists in the related field.

When measurements are listed as a result of a SIMBAD query, they are normally preceded by a header providing a very short title to each listed parameter.

The important rôle now played by the VIzIER database of catalogues (Ochsenbein et al. 2000), coming with easier interoperability of services, is changing the strategy for inclusion of observational measurements

\footnotetext{
${ }^{6}$ In the early times of the Catalog of Stellar Identifications (Ochsenbein et al. 1981), the Durchmusterung number had been used as a preferred name for stars.
}

Table 2. List of measurement types currently included in SIMBAD, together with the number of entries (October 1999)

\begin{tabular}{|c|c|c|}
\hline Name & Observational data & $\#$ \\
\hline CEL & Ultraviolet photometry from Celescope & 5230 \\
\hline $\mathrm{Cl} . \mathrm{G}$ & $\begin{array}{l}\text { Clusters of Galaxies (Abell \& Corwin } \\
\text { 1989) }\end{array}$ & 5345 \\
\hline Einstein & $\begin{array}{l}\text { Einstein Observatory Soft X-ray } \\
\text { Source List }\end{array}$ & 5668 \\
\hline GEN & $U B V B_{1} B_{2} V_{1} G$ Geneva photometry & 3650 \\
\hline GJ & $\begin{array}{l}\text { Absolute magnitudes and spatial veloc- } \\
\text { ities of nearby stars }\end{array}$ & 2368 \\
\hline Hbet & $\mathrm{H}_{\beta}$ index & 32278 \\
\hline HGAM & uivalent width & 723 \\
\hline IRAS & IRAS Point Source Catalog & 245784 \\
\hline IRC & $\begin{array}{l}\text { KI photometry from Two Micron Sky } \\
\text { Survey }\end{array}$ & 4880 \\
\hline IUE & $\begin{array}{l}\text { International Ultraviolet } \text { Explorer } \\
\text { (Merged Observation Log) }\end{array}$ & 66805 \\
\hline JP11 & $\begin{array}{l}U B V R I J K L M N H \text { 11-colour Johnson } \\
\text { photometry }\end{array}$ & 5892 \\
\hline MK & $\begin{array}{l}\text { Stellar spectral classification in } \\
\text { Morgan-Keenan system }\end{array}$ & 190231 \\
\hline oRV & $\begin{array}{l}\text { Stellar Radial velocities (also under } \\
\text { GCRV) }\end{array}$ & 68783 \\
\hline PLX & Trigonometric parallaxes & 16329 \\
\hline pm & $\begin{array}{l}\text { Proper motions (from various astro- } \\
\text { metric catalogues) }\end{array}$ & 243065 \\
\hline pos & $\begin{array}{l}\text { Positions (from various astrometric } \\
\text { catalogues) }\end{array}$ & 668953 \\
\hline ROT & Rotational velocities $(V \sin i)$ & 7181 \\
\hline RVEL & $\begin{array}{l}\text { Radial velocities of extragalactic ob- } \\
\text { jects }\end{array}$ & 36552 \\
\hline SAO & $\begin{array}{l}\text { Positions and proper motions from } \\
\text { SAO catalogue }\end{array}$ & 252384 \\
\hline TD1 & $\begin{array}{l}\text { Ultraviolet magnitudes from TD1 } \\
\text { satellite }\end{array}$ & 25972 \\
\hline UBV & Johnson $U B V$ photometry & 141215 \\
\hline uvby & Strömgren $u v b y$ photometry & 37986 \\
\hline $\mathrm{V} *$ & Data related to variable stars & 25764 \\
\hline$z$ & $\begin{array}{l}\text { Redshifts (of distant galaxies and } \\
\text { quasars) }\end{array}$ & 88888 \\
\hline
\end{tabular}

into SimBAD. Let us take the example of the Hipparcos and Tycho catalogues (ESA 1997): once the HIP or TYC identifier is available from SIMBAD it appears convenient enough to provide the user with a WWW link to the corresponding data in VizieR rather than overloading the SIMBAD database with the full Hipparcos and Tycho catalogues. This functionality is currently being implemented for important catalogues which have already been cross-identified.

As a complement, the WWW interface includes pointers to external archives, currently: the INES database of the IUE project (Rodriguez-Pascual et al. 1999); the highenergy observational archives at HEASARC (HEASARC team 1995). 


\subsection{Bibliographical references}

One of the key features of the SIMBAD astronomical database is the unique coverage of bibliographical references to objects. The bibliographic index contains references to stars from 1950 onwards, and to galaxies and all other objects outside the solar system from 1983 onwards. Presently (November 1999) there are about 3 million references taken from 110000 papers published in the 100 most important astronomical periodical publications.

\subsubsection{Bibliographical data}

Articles are scanned in their entirety, and references to all objects mentioned in the title, in the abstract, in the text, in the figures, or in the tables are included in the bibliography. Tables larger than 1000 objects are usually considered as catalogues and processed separately.

No assessment is made of the relevance of the citation in terms of astronomical contents: the paper can be entirely devoted to the object, or simply give a side mention of it - in both cases this gives a reference in SImBAD. Note that, for instance, the NED team (Helou et al. 1991) applies a different strategy when covering bibliography of extragalactic objects, and tends to select only those papers that appear most relevant. Clearly, SimBAD approach favours exhaustivity, at the cost of increased information noise.

A code (nicknamed bibcode) is assigned to each considered paper: this 19-digit bibcode contains in principle enough information to locate the article (including year of publication, journal, volume, page, etc.).

When one retrieves the bibliography of a SIMBAD object, a list of codes is usually given, and - according to the options used - these codes are automatically matched against a bibliographic file which provides the full reference, title and list of authors for each citation, together with an anchor pointing to the electronic version of the article.

Currently, in SimbaD, about $50 \%$ of the objects have no bibliographic reference. Among the most cited objects (more than 2000 references) are the Large Magellanic Cloud, M 31, 3C 273, and the supernova SN 1987A.

\subsubsection{Bibliographic reference coding convention}

The structure of the 19-digit bibcode has been defined in close collaboration with the NED group at NASA/IPAC so that both databases share the same coding system (Schmitz et al. 1995). It is also used, with some adjustments, by the Abstract Service of the Astrophysics Data System (ADS, Kurtz et al. 2000), and by the electronic journals (see e.g., Boyce \& Dalterio 1996). Reference codes have the following general structure:

\section{YYYYJJJJJVVVVMPPPPA}

YYYY Year of the publication.

JJJJJ Standard abbreviation for the periodical.

VVVV Volume number (for a journal) or, in the second character of this field, one of the following abbreviations for another publication: B (book), C (catalogue), $\mathrm{P}$ (preprint), R (report), $\mathrm{S}$ (symposium), $\mathrm{T}$ (thesis), $\mathrm{U}$ (unpublished).

M Specific qualifier for a paper:

L letter

$\mathrm{p}$ pink page (in MNRAS)

$\mathrm{a}-\mathrm{Z}$ issue number within a volume

A-K issue designation used by publisher

Q-Z to distinguish articles on the same page.

PPPP Page number.

A First letter of the first author's last name (or ":" if the first author cannot be identified).

Example: 1991A\&A...246L..24M for Astron. Astrophys. 246, L24, 1991, a Letter to the Editor of Astronomy \& Astrophysics, by Motch et al.

For a complete description see Schmitz et al. (1995), or the WWW server ${ }^{7}$.

\subsubsection{Comments in the references}

Several types of comments are associated with the references in SIMBAD and normally displayed after the reference:

- General comments: they are often comments added by the bibliographers, about the problems encountered while cross-identifying the objects mentioned in the paper, typos in object names, etc.

- Notes about the existence of associated electronic tables, or abstracts in the CDS server. Papers including no object are also flagged.

- Information on how the quoted objects are named in Simbad (comments related to the Dictionary of Nomenclature of Celestial Objects).

\subsection{Statistical aspects of the Data Contents}

The astronomical content of SIMBAD results from the complex process of folding into the database a selection of important catalogues, and of surveying the complete astronomical literature.

This can be illustrated by the histogram in $V$ magnitudes of Fig. 1. The coverage is reasonably complete up to beyond magnitude 10 for stars, after the inclusion of the Tycho catalogue. Many objects in the range 12 to 26 th mag come from extensive studies of objects in selected sky areas: deep fields, external galaxies, etc.

\footnotetext{
7 http://cdsweb.u-strasbg.fr/simbad/refcode.html
} 


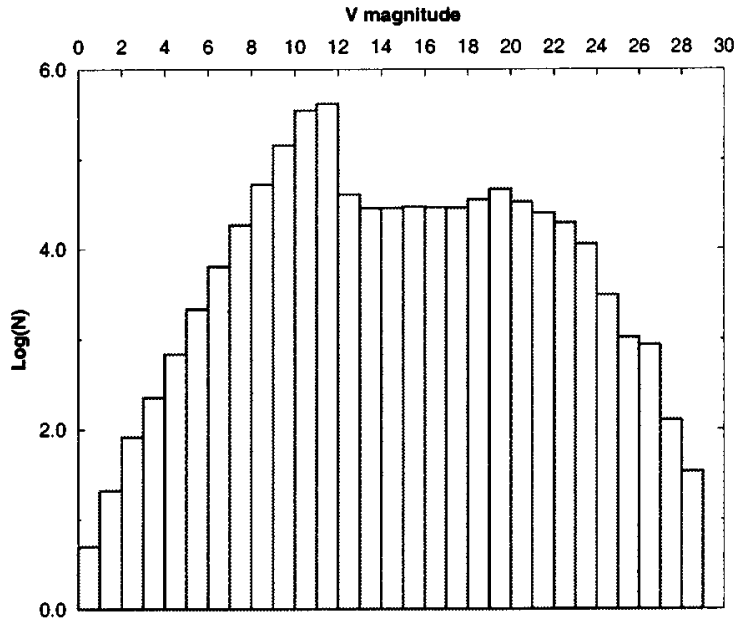

Fig. 1. Histogram of the number of objects in SIMBAD per magnitude interval ( $V$ magnitude; logarithmic scale)

Some well-known very large catalogues are not part of SimBAD: for instance the Hubble Telescope Guide Star Catalogue (GSC, Lasker et al. 1990) is not systematically included (even if GSC identifiers have been added for all Tycho stars present in SIMBAD). This results from a compromise aiming to save database load as well as manpower for cross-identification and quality control. Note that ViziER and ALAdin give access to the full GSC catalogue (and to even larger catalogues and databases such as USNO-A, DENIS, 2MASS).

Figure 2 illustrates the increase of the data contents of the database in the years 1990 to 1999.

\section{SIMBAD structure and query management}

SimBAD query mechanism can be summarized by the following key features:

- Database queries can be made mainly through:

- identifiers (names of astronomical objects) and lists of identifiers;

- sets of coordinates (retrieving one object by its position on the sky, or extracting all objects lying in a given direction), and;

- sampling criteria (or filters);

- Data output is driven by formats. The user may define his/her own formats or modify existing ones. Output files can be saved and mailed to the user;

- The user interface is adaptable to user preferences.

The database management system of SIMBAD has been developed by the CDS, using the concepts of object-oriented programming.

\subsection{Object-oriented concepts}

The command language is using the concepts of objects (or agents). Typical object classes are: astronomical object,

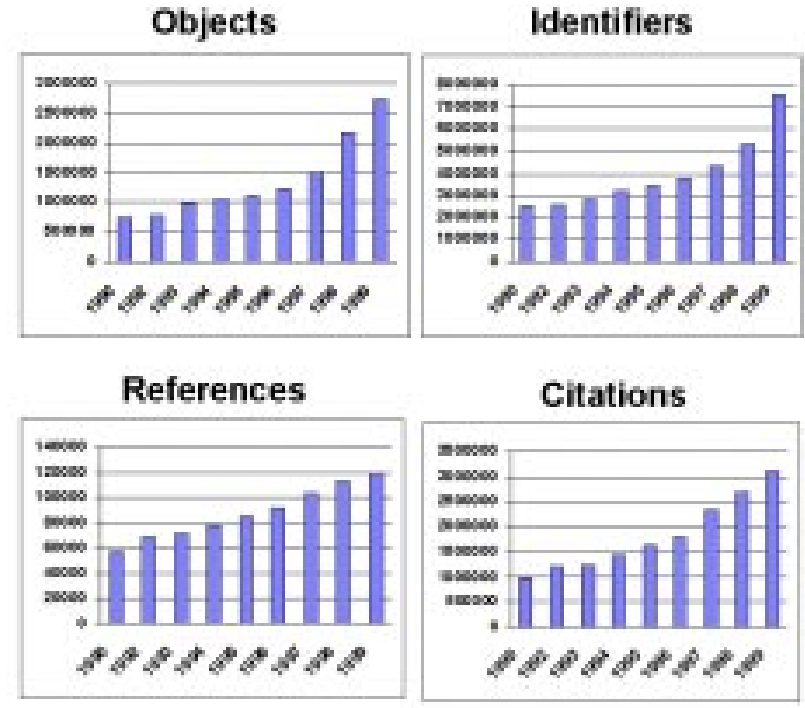

Fig. 2. Histograms of different types of database entries for the years 1990 to 1999: number of objects (top left), number of identifiers (top right), number of references (bottom left), number of citations (bottom right). The numbers given are the total numbers of entries present in the database at the end of the corresponding year

object list, database, session, reference list, filter, format. Examples of methods are: display, describe, bye (quit).

This structure is only visible for the user of the command line interface. The WWW interface is rendered quite independent of the database structure.

\subsection{Indexing}

SIMBAD is organized for optimized access by identifier (through an index table of object names) and by position, through an index of small regions.

Identifiers: A B-tree file contains all identifiers allowing a fast access to any of them. For each identifier, a record contains a pointer to the astronomical object itself in the main database.

Position: Indexing by coordinates is done in two steps: the coordinates are mapped into a set of boxes. SIMBAD uses the spherical-cubic projection - a technique also used, e.g., for the Cosmic Background Explorer (COBE) data: the celestial sphere is projected onto the six faces of a cube, giving six boxes at the first level. By dividing each face into four parts, one obtains a partition at level two. Further levels are obtained by further divisions of each box into four sub-boxes. Through this mechanism one obtains 6144 boxes at the level 5 with an average size of 6 square degrees and an average number of objects of 500. Box \#6145 contains all objects without recorded position. 
In order to optimize access to objects in a coordinate box, all objects belonging to a box should be physically grouped in a common place in the database. This is done through a clustering mechanism placing objects from the same box in data blocks linked together in the database files.

When a set of criteria includes some limitations in coordinates, this generates the definition of a list of boxes including the requested area: all entries from these boxes are read and checked against the whole set of criteria.

When a set of criteria includes no limits in sky position, the complete database must be scanned - a long and somewhat expensive operation, which takes typically 15 minutes in the current hardware configuration.

\subsection{Query by identifier}

In principle any name found in the literature - provided it is given as a syntactically correct character string - can be submitted to the database in order to retrieve information known for this object.

The general syntax of an identifier is the abbreviated catalogue name (or acronym: generally one to four characters), followed by a number or a name (character string) within the catalogue.

Object names such as Vega and Altair, but also Barnard's star, Crab Nebula, Sgr A, HDFN, or HDFS are stored in the database in a specific catalog called "NAME", while star names in constellations, such as $\alpha$ Lyrae, are stored in the catalogue "*", and variable stars (such as RR Lyrae) in the catalogue "VAR" (also called "V*").

The user can generally type Vega, Altair, alf Lyrae (or alf Lyr): the sesame name resolving module (Sect. 8.2) is used for guessing the catalogue and making the internal conversion. There are however some difficult cases in which the NAME keyword remains necessary, such as in NAME SGR $1900+14$ where SGR stands for Soft Gamma Repeater.

In addition the following hints can help the user understand the best way to submit an identifier to SimbaD:

Case sensitivity: SIMBAD is not case-sensitive at this level: ALF AQL or alf Aql are, for instance, both valid. There are some exceptions to the rule, such as the cases of the star cluster RMC 136a, or the star in a multiple system $\mathrm{VdBH} 25 \mathrm{a} \mathrm{A}$, for which case-sensitivity may be necessary for solving format ambiguities.

Greek letters: should be abbreviated as three letters: alf, bet, for $\alpha$ and $\beta$, but also mu. nu. and pi. (with a dot), for $\mu, \nu$ and $\pi$.

Constellations: constellation names should be abbreviated with the usual three letters: alf Boo, del Sct, FG Sge, NOVA Her 1991. The full list is available on-line ${ }^{8}$.

8 http://simbad.u-strasbg.fr/guide/chB.htx
Multiple systems: Identifiers of a multiple system may generate a list of the objects of the system. For instance, ADS 5423 calls for the four components, A to $\mathrm{D}$, of the stellar system around Sirius. This is true only for some specific identifiers.

Stellar clusters: Clusters which have no NGC or IC number are named under the generic appellation Cl followed by the cluster name and number: e.g., $\mathrm{Cl}$ Blanco 1 is the 1st stellar cluster named by Blanco. Stars in clusters may belong to a "main" designation list, or to subsequent lists. NGC 5272692 is star 692 in the list by Von Zeipel, considered as the main list. Subsequent lists have designations starting with $\mathrm{Cl} *$. Examples: Cl* NGC 5272 AC 968 (list by Auriere \& Cordoni); $\mathrm{Cl}^{*}$ Melotte $25 \mathrm{VA} 13$ (13th star in the list by Van Altena for Melotte 25 - the Hyades cluster); $\mathrm{Cl}^{*}$ Collinder 110 DI 1101 (list by Dawson \& Ianna there is no "main" list for this cluster). More details are available in the on-line description ${ }^{9}$.

Unknown name ?: If the object name seems unknown to SIMBAD, the user is advised to enter the coordinates of the object: the object may actually exist in the database under a different designation. Submitting the identifier, or the name of the first author of the catalogue, to the Dictionary of Nomenclature may also give useful clues.

Figure 3 illustrates the response received from the database after submitting the identifier "M 81". In the identifier list, the meaning of acronyms, such as [VDD93], is explained through a link to the on-line Dictionary of Nomenclature.

The user interface provides an option for querying around objects, with a radius set by default at $10^{\prime}$. This is equivalent to a query by position using the object coordinates.

It is also possible to generate the list of 10 or 25 next objects following a given identifier, or to submit a list of object names, stored in a file with one identifier per line.

\subsection{Query by coordinates}

Query by coordinates can be used to retrieve all objects in a circular field defined by the coordinates of the center and a radius.

The coordinates can be replaced by the name of an object lying at the center of the field, in which case the coordinates are found through an internal query to SiMBAD. The radius can have any size (default value is $10^{\prime}$ ). Queries with a radius smaller than $1-2^{\circ}$ are answered quite instantaneously.

${ }^{9}$ http://simbad.u-strasbg.fr/guide/chC.htx 


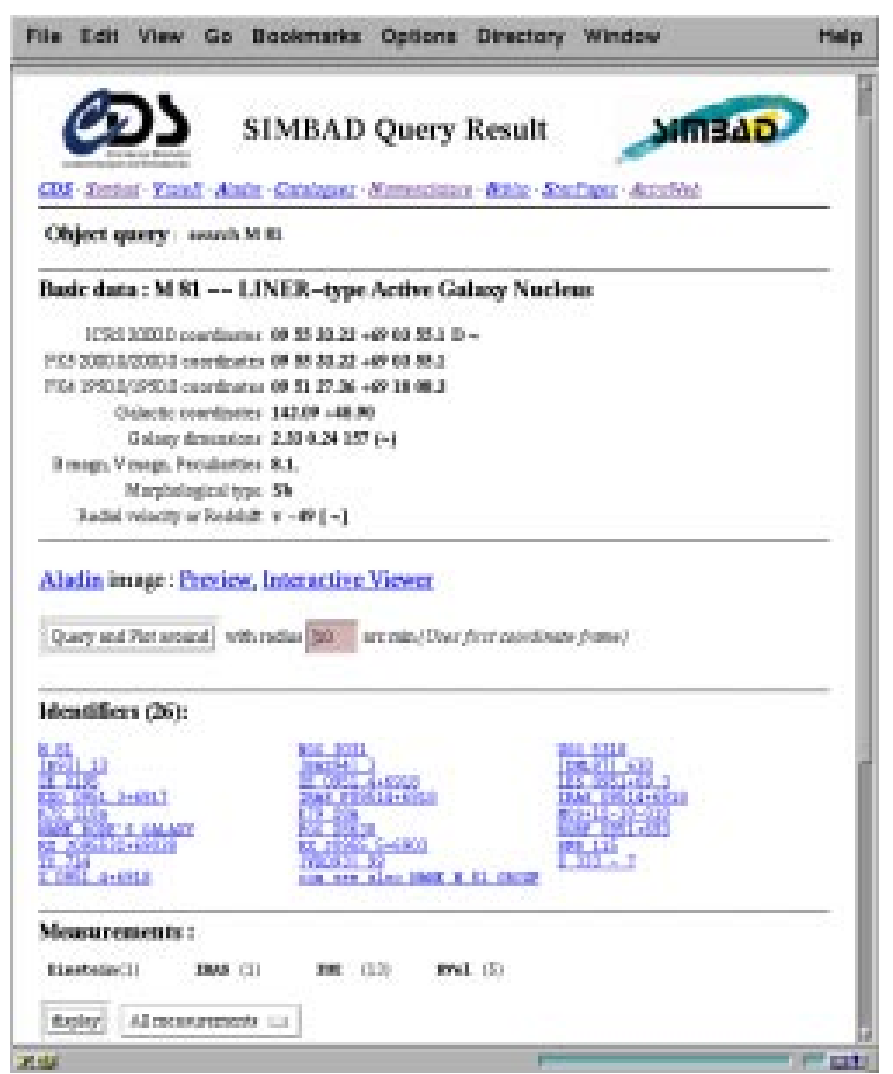

Fig. 3. Example of Simbad response page for a query concerning M 81 (only the first part of the response is visible here)

\subsection{Sampling}

The sampling mode (also named filter) allows users to define criteria for selecting objects in SIMBAD.

The user may extract objects which satisfy one set of coordinate criteria, several physical criteria (using a simple syntax), objects which have specified identifiers or measurements, and, finally, objects having citations within a range of years.

The WWW interface provides an interactive form which presents all possible sampling options.

The resulting list may be ordered according to sort criteria and, furthermore, it is possible, through the command line mode, to define precisely the output format.

Note that reading the whole database for extracting a sample spread on the whole celestial sphere is possible, but quite time-consuming (as mentioned above). The user is thus encouraged to test the filter on a limited region of the sky, before applying it to the whole database.

\subsection{Charts and sky maps}

After a sampling by position the user can ask for the corresponding sky plot. This feature is only available through the WWW interface and is generally optimized for a radius range of $10-60$ minutes.

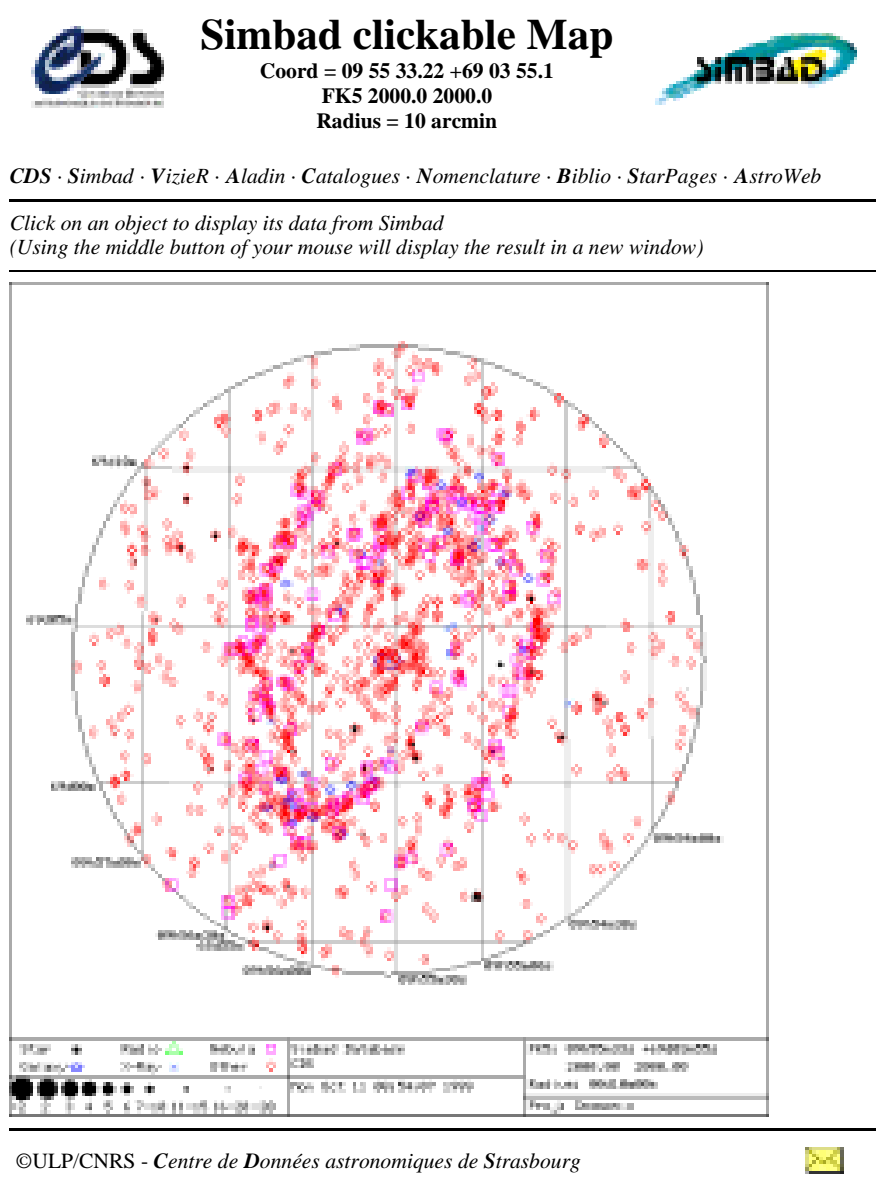

Fig. 4. Example of finding chart around M 81 (radius of the circular field: $10^{\prime}$ )

The maps display the objects with different symbols according to object type; symbol size for stars varies with object magnitude (see Fig. 4). The maps are clickable and return the object in Simbad corresponding to cursor position.

The WWW interface provides also direct access to the Aladin interactive digitized atlas (Bonnarel et al. 2000) as illustrated in Fig. 5.

\subsection{Batch mode}

SIMBAD can be queried in batch mode, by submitting a mail to the special address

smbmail@simbad.u-strasbg.fr.

This is especially useful in case of poor interactive connectivity, or for submitting time-consuming queries or lists. A WWW form ${ }^{10}$ helps to prepare the submission.

10 http://cdsweb.u-strasbg.fr/simbad/batch.html 


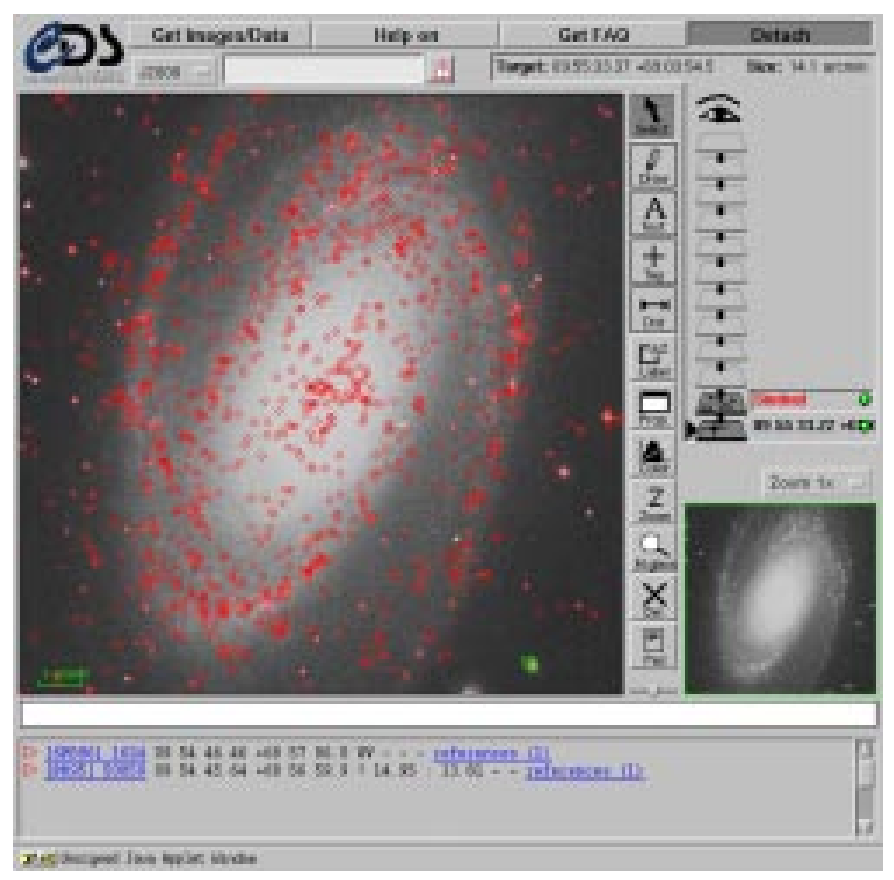

Fig. 5. Use of Aladin for displaying Simbad entries (red diamonds) on top of a DSS-I/STScI image around M 81 (width of the field: $14.1^{\prime}$ )

\subsection{Resolving a bibliographical reference code}

It is possible to obtain a complete bibliographical reference, by entering the corresponding reference code (bibcode).

A reference code can be supplied without indicating all the fields: the first reference corresponding to the truncated code will be displayed. An ampersand (\&) should be added at the end of the truncated bibcode.

\subsection{Additional tools}

Additional tools include special commands for querying auxiliary databases, on-line help, log files, etc. More details can be found in the SIMBAD User's Guide or on the Web pages.

\section{User interfaces to SIMBAD}

There are several user interfaces to SIMBAD. New users are advised to go directly to the WWW interface, unless they have very specific needs.

- The Web interface is currently the easiest access mode to Simbad. This interface takes benefit of the WWW features to provide the user with additional links to internal documentation, associated services (ALADIN, VIZIER), and external archives (currently: the INES database of the IUE project; the high-energy observational archives at HEASARC). Some features, such as the finding chart, have been specifically designed for the Web and are not available through the other modes.

- The command line interface is the basic underlying interface to the database, which serves as a basis for the other more user-friendly interfaces. During many years it was the sole access mode to the database, and many users who are accustomed to the commands may find it quicker and more versatile. It implies a remote login (through telnet) on the simbad machine in Strasbourg, and the user needs to have a user name and password (see Sect. 6.1).

- A graphical interactive user interface to SimBAD, XSImBAD, taking benefit of the $\mathrm{X}$ Window environment has been developed in 1993-94 for distribution to users working in a Unix environment. It is now obsolete, because all the functionalities, and additional ones, are more easily available through the Web.

- A client/server package is distributed on request to data managers of archives and information systems, when they need to organize the most efficient access to Simbad for the resolution of object names into the corresponding position, or the retrieval of other information provided by the database, such as the reference list for a given object. Written currently in C language, it can easily be plugged into any application able to access $\mathrm{C}$ routines. Distribution is subject to CDS approval.

\section{SIMBAD usage}

\subsection{Charging policy}

SImBAD is a charged service. The telnet access is protected by userid/password, and the WWW access is protected either by IP address or by password.

Users have to register, and get a userid/password from the CDS staff (or from the U.S. agent for American users).

In the U.S. there is no invoicing for the end-user because the charges are covered globally by NASA for all U.S. users.

In Europe, the same situation is also true for users from ESO and ESA member states, thanks to an agreement signed with European Southern Observatory and ESA Space Telescope European Coordinating Facility (since January 1995).

Special arrangements also exist or are currently being negotiated with other countries, including Canada, Australia, and Japan.

\subsection{Usage statistics}

There are currently (November 1999) some 7000 user accounts from 64 different countries. The development of the WWW access makes difficult to keep precise track 
of the individual usage statistics, but the global statistics show that the world wide interest for accessing the SIMBAD database continues to increase regularly over the years.

The number of SIMBAD queries evolved from about 30000 per month in 1997 to about 100000 per month in 1999. About $50 \%$ of the queries come through the client/server mode.

A mirror copy of SIMBAD has been established at CfA (Harvard) for convenience of US users, and about one third of the queries are currently processed on the mirror site (including name resolving activities for the ADS and major US NASA archives). The mirror copy is managed by CDS and updated every night.

\section{SIMBAD updates and quality control}

\subsection{Updating SIMBAD}

SIMBAD is kept up-to-date on a daily basis, as the result of the collaboration of the CDS team, in Strasbourg, with bibliographers in Observatoire de Paris (DASGAL), Institut d'Astrophysique de Paris, and Observatoire de Bordeaux (Laloë et al. 1993; Laloë 1995) who systematically scan the articles published in some 100 astronomy journals.

The references are updated very soon after reception of the journal issues, and in some cases directly from the journal table of contents, through agreements with the Editors. New data concerning the objects (identifiers, basic data), and new acronyms for catalogs or tables are being entered when appropriate.

The inclusion of a large catalogue in the database is often a long-term task which may span over several months or years; the collaboration of specialists in the different fields is systematically sought.

The improvement of the SIMBAD astronomical contents relies on a network of collaborations: a list of the main current contributors is given in Table 3. More generally, help of other contributing institutes and authors, too numerous to be cited here, is gratefully acknowledged.

\subsection{Quality control}

The data contained in SIMBAD are also permanently updated, as a result of errata, remarks from the bibliographers (during the scanning of the literature), integration of lists and catalogues, quality controls, or special efforts initiated by the CDS team to better cover some specific domains (e.g., multi-wavelength emitters and complex objects).

Requests for corrections, errata, or suggestions are regularly received from SIMBAD users through a dedicated hot line, at e-mail address question@simbad.u-strasbg.fr. A few dozens of messages are usually received every week,
Table 3. Main institutes associated to the CDS for improving the data contents of SIMBAD

\begin{tabular}{|c|c|}
\hline Bibliography & $\begin{array}{l}\text { Observatoire de Paris, } \\
\text { Institut d'Astrophysique de Paris, } \\
\text { and Observatoire de Bordeaux }\end{array}$ \\
\hline Astron. contents & GRAAL, Montpellier \\
\hline Galaxies & $\begin{array}{l}\text { Observatoire Midi-Pyrénées and } \\
\text { NASA/IPAC Extragalactic Database }\end{array}$ \\
\hline Photometry & $\begin{array}{l}\text { Observatoire de Genève and Institut } \\
\text { d'Astronomie de Lausanne }\end{array}$ \\
\hline Astrometry & $\begin{array}{l}\text { Astronomisches Rechen Institut, } \\
\text { Heidelberg }\end{array}$ \\
\hline Binary stars & Observatoire de Besançon \\
\hline High-energy & Observatoire de Strasbourg \\
\hline
\end{tabular}

and processed on a daily basis by the member of the team who is on duty for that week, or transmitted to the key person in case of specialized questions. Remarks received from the users by this way are especially welcome, as they help the CDS team to improve the database contents through the scrutiny of specialists' eyes.

Developing new tools for quality control of the database is a major challenge for the future, and CDS is exploring possible solutions. Multivariate analysis applied to bibliographic information retrieval has been proven a possible tool for developing quality control in a database such as SimbAD (Lesteven 1995).

\subsection{Towards automation of updating procedures}

The advent of electronic publishing brings new perspectives for improvement and automation of the updating procedures.

In a first place, tables of contents of the major journals are now received electronically through the network, thanks to journal Editors and Publishers, thus reducing the risk of errors. Regularly, a number of electronic lists of objects are also folded into SIMBAD through semiautomatic procedures. The next step will be the automatic flagging of object names in the text of the articles: this has now become a very interesting medium-term goal.

Two ways of achieving this flagging are currently being considered:

- the first one is to ask the authors, with the help of the Editors of electronic journals, to flag astronomical object names in their text; this can be done, for instance, by the use of a lobject\{ \} command within the $\mathrm{TEX}$ or $\mathrm{LAT}_{\mathrm{E} X}$ source, which will be eventually used to build an anchor pointing towards SimbAD, or another database, in the on-line version made available on the network. This approach has been adopted by the Editors of Astronomy \&6 Astrophysics; 
- another approach is the use of intelligent search tools for identifying object names within the electronic version of the paper, using a set of syntactic and semantic rules, and the Dictionary of Nomenclature as a reference database for already known objects.

The first approach seems safer, provided the authors understand what exactly they are being required, and accept this (minor) additional work load. The latter implies a lot of fine tuning from the system developers. The current experience with the handling of publications (Lesteven et al. 1998) suggests that both approaches may be needed, and that a careful quality control, including final check by an expert, will probably remain necessary to avoid errors or misinterpretations, and to ensure appropriate completeness.

\section{Nomenclature}

\subsection{The dictionary of nomenclature}

Designations of astronomical objects are often confusing. A complete list of astronomical designations has been collected and published by Lortet et al. (1994) in the Dictionary of Nomenclature of Celestial Objects outside the Solar System.

This information is available on-line through the info command, or on the $\mathrm{WWW}^{11}$. This service is the electronic look-up version of the Dictionary which is now under the responsibility of CDS. It is kept up-to-date on a weekly basis; about 15 new acronyms are incorporated every week.

The Dictionary currently provides full references and usages about some 5000 different acronyms. It is used by the International Astronomical Union as a reference for its recommendations related to nomenclature.

\subsection{The sesame module}

The sesame module is used inside SIMBAD for the management of possible variations in the naming of astronomical objects. It is based on a list of rules, written as regular expressions, allowing translation of the submitted name into its SIMBAD canonical form; it is only made visible to the user when a message mentions the submitted syntax and its translation.

There are cases where ambiguities cannot be solved. This is actually specific to the broad context of SimBAD. Let us give an example: in the context of extragalactic objects "N" is a possible abbreviation for "NGC" (accepted by NED); but people studying Novae would frequently use "N" as an abbreviation for Nova, people studying H II regions would use it for naming nebulae in the Magellanic Clouds (LHA 120-N or LHA 115-N), and "N" has also

\footnotetext{
11 http://vizier.u-strasbg.fr/cgi-bin/Dic-Simbad
}

been found in the literature for cluster stars studied by Nordlund in NGC 2099 (Cl* NGC 2099 N), for stars studied by Neckel ([N78]), or even for "New" parts of the galaxy NGC 1275 ([NJS93] in SimBAD). When a name like "N 1992" is submitted to SIMBAD the ambiguity cannot be solved without requesting additional information from the user.

\section{Integration of SIMBAD into the CDS hub}

While the CDS databases have followed different development paths, the need to build a transparent access to the whole set of CDS services has become more and more obvious with the easy navigation permitted by hypertext tools (Genova et al. 2000). ALADIN has become the prototype of such a development, by giving comprehensive simultaneous access to SimbaD, the VizIER Catalogue service, and to external databases such as NED, using a client/server approach and, when possible, standardized query syntax and formats.

In order to be able to go further, the CDS has built a general data exchange model, taking into account all types of information available at the Data Center, known under the acronym of GLU for Générateur de Liens Uniformes - Uniform Link Generator (Fernique et al. 1998).

In the current stage of development, the WWW interface to SIMBAD provides access to ALADIN previewer (reference image around one object), and to the AladiN interactive Java program (see Bonnarel et al. 2000). There are also links between SIMBAD and the bibliographic services developed or mirrored at CDS, and more generally to the ADS and the electronic journals.

While this article is written stronger links between SIMBAD and VIZIER are just being created allowing even easier transfers of data and information between both services. This will also make easier to build new links pointing to distributed data archives, beyond those already existing (currently: IUE/INES and HEASARC).

\section{Future developments}

In the near future, the CDS team expects to go on enriching the database contents and system functionality. The users play an important role in that respect, by giving feedback on the desired features, on the user-friendliness of the interfaces, etc.

In the context of interoperability of distributed services, as currently discussed within the ISAIA project (Interoperable Systems for Archival Information Access; Hanisch 2000), SIMBAD is prepared to deliver resource profiles and to format the query outputs in a standard way, for instance XML (Ochsenbein et al. 2000).

As larger and larger astronomical datasets are being produced, the CDS is studying the concepts of a new generation database of several billion objects, instead of the 
current several million objects. We expect SIMBAD to remain an essential tool for astronomical research in the years to come.

Acknowledgements. CDS acknowledges the support of INSUCNRS, the Centre National d'Études Spatiales (CNES), and Université Louis Pasteur (ULP, Strasbourg). Many of the current developments of SIMBAD have been made possible by longterm support from NASA, ESA, and ESO. We thank more specifically J. Mead and G. Riegler (NASA), P. Benvenuti (ESA/ST-ECF), and P. Quinn (ESO) for their help in setting up the current agreements.

Developing and maintaining the database is a collective undertaking to which many contributors - too numerous to be listed here - are associated. A special mention shall be made of M.-J. Wagner, F. Woelfel, J. Marcout (Strasbourg), A. Beyneix, G. Chassagnard (IAP, Paris), N. Ralite, S. Pasquier (Bordeaux), E. Davoust (Toulouse), and B. Skiff (Lowell Observatory), who are watching with great care over the SimBAD contents.

We want finally to thank Jean Delhaye, Jean Jung, Carlos Jaschek and Michel Crézé for their leadership and their vision in the consecutive early phases of the SIMBAD project.

\section{References}

Abell G.O., Corwin H.G. Jr., Olowin R.P., 1989, ApJS 70, 1 (ABCG)

Bonnarel F., Fernique P., Bienaymé O., et al., 2000, A\&AS 143, 33 (Aladin)

Boyce P., Dalterio H., 1996, Phys. Today 49, 42

Dubois P., Ochsenbein F., Paturel G., 1983, Bull. Inform. CDS 24,125

Egret D., 1983, Bull. Inform. CDS 24, 109 (SIMBAD Story)

Egret D., Crézé M., Bonnarel F., et al., 1995, in Information \& On-line Data in Astronomy, Egret \& Albrecht (eds.). Kluwer Academic Publ., p. 163

Egret D., Wenger M., Dubois P., 1991, in Databases and Online Data in Astronomy, Albrecht M.A. \& Egret D. (eds.). Kluwer Academic Publishers, p. 79

ESA, 1997, The Hipparcos and Tycho Catalogues, ESA SP1200

Feissel M., Mignard F., 1998, A\&A 331, L33 (ICRS)

Fernique P., Ochsenbein F., Wenger M., 1998, in Astronomical Data Analysis Software and Systems VII, ASP Conf. Ser. 145, 466 (GLU)

Genova F., Bartlett J.G., Bienaymé O., et al., 1996, Vistas Astron. 40, 429

Genova F., Bartlett J.G., Bonnarel F., et al., 1998, in Astronomical Data Analysis Software and Systems VII, ASP Conf. Ser. 145, 470
Genova F., Egret D., Bienaymé O., et al., 2000, A\&AS 143, 1 (CDS)

Hanisch R., 2000, Computer Physics Communications (in press)

Heck A., Egret D., 1987, Messenger 48, 22-24

HEASARC team, 1995, in Information \& On-line Data in Astronomy, Egret \& Albrecht (eds.). Kluwer Academic Publ., p. 139

Helou G., Madore B.F., Schmitz M., et al., 1991, in Databases \& On-line Data in Astronomy, Albrecht \& Egret (eds.). Kluwer Acad. Publ., p. 89

Høg E., Kuzmin A., Bastian U., et al., 1998, A\&A 335, 65 (TRC)

Houk N., Cowley A.P., 1975, Michigan Catalogue of TwoDimensional Spectral Types for the HD Stars, Vol. 1 (MK)

Jaschek M., 1978, Bull. Inf. CDS 15, 121

Kurtz M., Eichhorn G., Accomazzi A., et al., 2000, A\&AS 143, 41 (ADS)

Laloë S., 1995, in Proceedings of the LISA-II Conference, Vistas Astron. 39, 179

Laloë S., Beyneix A., Borde S., et al., 1993, CDS Inform. Bull. 43,57

Lasker B.M., Sturch C.R., McLean B.J., et al., 1990, AJ 99, 2019 (GSC)

Lesteven S., 1995, Vistas Astron. 39, 187

Lesteven S., Bonnarel F., Dubois P., 1998, in Proceedings LISA III Conference, Grothkpof U., et al. (eds.), ASP Conf. Ser. 153, 61

Lortet M.C., Borde S., Ochsenbein F., 1994, A\&AS 107, 193

Mermilliod J.-C., 1987, A\&AS 71, 413 (UBV)

Nilson P., 1973, Uppsala General Catalogue of Galaxies (UGC)

Ochsenbein F., 1982, in Automated Data Retrieval in Astronomy, Jaschek C. \& Heintz W.D. (eds.), IAU Coll. 64. Dordrecht, D. Reidel Publishing Company, p. 171 (BSI)

Ochsenbein F., Albrecht M., Brighton A., et al., 2000, in Astronomical Data Analysis Software and Systems IX, ASP Conf. Ser. (in press) (XML)

Ochsenbein F., Bauer P., Marcout J., 2000, A\&AS 143, 23 (VizieR)

Ochsenbein F., Bischoff M., Egret D., 1981, A\&AS 43, 259 (CSI)

Ochsenbein F., Dubois P., 1992, in Astronomy from Large Databases, II, Heck A. \& Murtagh F. (eds.), p. 405

Rodriguez-Pascual P.M., González-Riestra R., Schartel N., Wamsteker W., 1999, A\&AS 139, 183 (INES)

Schmitz M., Helou G., Dubois P., et al., 1995, in Information \& On-line Data in Astronomy, Egret \& Albrecht (eds.). Kluwer Acad. Publ., p. 259

Vorontsov-Velyaminov B., et al., 1962, Morphological Catalogue of Galaxies, in 5 parts, Moscow State University, Moscow (MCG) 